\title{
BOOK-TAX DIFFERENCES E GERENCIAMENTO DE RESULTADOS NO MERCADO DE AÇÕES DO BRASIL
}

\author{
BOOK-TAX DIFFERENCES AND EARNINGS MANAGEMENT: EVIDENCE IN THE BRAZILIAN \\ EQUITY MARKET
}

BOOK-TAX DIFFERENCES Y GESTIÓN DE RESULTADOS EN EL MERCADO DE ACCIONES DE BRASIL

\section{RESUMO}

Este estudo verifica a relação entre book-tax differences (BTD) e gerenciamento de resultado em companhias listadas na BM\&FBovespa no período de 2005 a 2009. Metodologicamente, foram empregadas duas abordagens: (i) distribuição de frequências e (ii) accruals discricionários - Modelo Jones Modificado. Os achados indicam uma relação diretamente proporcional entre a BTD e os accruals discricionários. Foram identificadas evidências de que as entidades preponderantemente gerenciam seus resultados na mesma direção do sinal observado da BTD, além de buscarem apresentar o montante de BTD em nível e em variação em torno do ponto zero e desta forma evitar sinalizar baixa qualidade do lucro. Adicionalmente, foram encontradas evidências de que o tamanho da firma e a adoção do regime tributário de transição estão relacionados de forma inversamente proporcional ao nível dos accruals discricionários.

PALAVRAS-CHAVE Book-tax differences, gerenciamento de resultados, acumulações discricionárias, qualidade do lucro, mercado de capitais

Felipe Ramos Ferreira felipe.ramos.ferreira@gmail.com

Doutorando em Ciências Contábeis pelo Programa de Pós-Graduação em Ciências Contábeis, Fundação Instituto Capixaba de Pesquisas em Contabilidade, Economia e Finanças - Vitória - ES, Brasil

Antonio Lopo Martinez lopo@fucape.br

Professor do Programa de Pós-Graduação em Ciências Contábeis, Fundação Instituto Capixaba de Pesquisas em Contabilidade, Economia e Finanças - Vitória - ES, Brasil

Fábio Moraes da Costa fabio@fucape.br

Professor do Programa de Pós-Graduação em Ciências Contábeis, Fundação Instituto Capixaba de Pesquisas em Contabilidade, Economia e Finanças - Vitória - ES, Brasil

Renato Rovetta Passamani renato.passamani@bol.com.br

Mestre em Ciencias Contábeis pelo Programa de Pós-Graduação em Ciências Contábeis, Fundação Instituto Capixaba de Pesquisas em Contabilidade, Economia e Finanças - Vitória - ES, Brasil

Abstract This study aimed to investigate the relationship between book-tax differences (BTD) and earnings management in firms listed on the BM\&F Bovespa. The final sample consisted of 485 observations (firm-year) and the analysis included the period from 2005 to 2009. Methodologically, two approaches were employed in this study: (i) frequency distribution and (ii) discretionary accruals - Modified Jones Model. The findings indicate a directly proportional relationship between BTD and discretionary accruals. There was evidence that the firms manage their earnings in the same direction of the observed signal of BTD, and seek to present the amount of BTD in level and variation around the breakeven and thus avoid a relationship with low earnings quality. Additionally, evidence indicate that the size of firm and the adoption of the tax basis transition are inversely related to the level of discretionary accruals.

Keywords Book-tax differences, earnings management, discretionary accruals, earnings quality, equity market.

Resumen Este estudio verifica la relación entre book-tax differences (BTD) y gestión de resultados en compañías listadas en la BM\&FBovespa, en el período de 2005 a 2009. Metodológicamente, fueron empleados dos abordajes: (i) distribución de frecuencias y (ii) accruals discrecionales, Modelo Jones Modificado. Los resultados indican una relación directamente proporcional entre la BTD y los accruals discrecionales. Fueron identificadas evidencias de que las entidades preponderantemente gestionan sus resultados hacia misma dirección de la señal observada de la BTD, además de buscar presentar el monto de BTD en nivel y en variación en torno del punto cero y asi evitar señalizar baja calidad del lucro. Adicionalmente, fueron encontradas evidencias de que el tamaño de la empresa y la adopción del régimen tributario de transición están relacionados de forma inversamente proporcional al nivel de los accruals discrecionales.

Palabras clave Book-tax differences, gestión de resultados, acumulaciones discrecionales, calidad del lucro, mercado de capitales. 


\section{INTRODUÇÃO}

Dois temas recorrentes na literatura contábil nas últimas décadas são gerenciamento de resultado (earnings management) e a divergência existente entre o lucro contábil e lucro tributável (book-tax differences-BTD). Um dos motivos para a grande quantidade de pesquisas empíricas nestas duas áreas se deve à relevância dos dois tipos de usuários da informação contábil vinculados a cada tema, acionistas e governo (SHACKELFORD e SHEVLIN, 2001; HANLON e HEITZMAN, 2010; GRAHAM, RAEDY e SHACKELFORD, 2012).

O gerenciamento de resultado usualmente está associado a práticas discricionárias adotadas pelo gestor no intuito de manipular a informação contábil em consonância com algum tipo de incentivo econômico, ao passo que a BTD basicamente está relacionada a diferenças existentes entre a norma contábil (GAAP) e a norma tributária (por exemplo: Regulamento do Imposto de Renda).

O Brasil, recentemente, sofreu duas alterações na legislação societária no intuito de aproximar cada vez mais as normas contábeis brasileiras das normas contábeis internacionais. Nesta direção, tanto a Lei n. 11.638/07 como a n. 11.941/09 introduziram uma maior flexibilidade para o reconhecimento, mensuração e evidenciação da informação contábil, bem como uma menor influência dos efeitos tributários sobre a questão societária.

Diante do exposto, o objetivo desta pesquisa é verificar a relação entre BTD e o gerenciamento de resultado nas companhias listadas na BM\&F Bovespa. Por hipótese, acredita-se que a BTD pode ser parcialmente explicada por práticas oportunistas das empresas orientadas a gerenciar resultados contábeis. Metodologicamente, foram empregadas duas abordagens no estudo: (i) distribuição de frequências da BTD em nível e em variação e (ii) estimação dos accruals discricionários, ambas as técnicas com o objetivo de identificar a relação entre o gerenciamento de resultado e a BTD. Registre-se, por pertinente, que o Modelo Jones Modificado (DECHOW, SLOAN e SWEENEY, 1995) foi escolhido para estimar as acumulações discricionárias, por ser amplamente utilizado em pesquisas desta natureza (AYERS, JIANG e YEUNG, 2006; PHILLIPS, PINCUS, REGO, 2003; RONEN e YAARI, 2008).

Apesar da existência de uma vasta literatura sobre os temas gerenciamento de resultado e book-tax difference, este estudo oferece novos insights para se entender como as práticas de earnings management estão associadas ao planejamento tributário, que emergem no Brasil com ca- racterísticas institucionais muito específicas. Os achados trazem importantes implicações para reguladores, auditores, investidores e demais usuários das informações contábeis. A BTD pode ajudar investidores a estimar e avaliar a qualidade das demonstrações financeiras e as autoridades reguladoras a realizar uma supervisão mais orientada de acordo com o perfil da BTD das firmas.

Para tanto, este artigo é apresentado com base na seguinte estrutura: na próxima secção é apresentado um breve referencial teórico, contemplando os principais achados da literatura sobre gerenciamento de resultado e BTD, seguido da apresentação da metodologia de pesquisa, resultados e conclusões.

\section{QUALIDADE DO LUCRO E GERENCIAMENTO DE RESULTADO}

Dechow, Ge e Schrand (2010) realizaram uma vasta revisão na literatura sobre a qualidade do lucro (earnings quality) envolvendo proxies, determinantes e consequências, e propuseram a seguinte definição para o termo: "higher quality earnings provide more information about the features of a firm's financial performance that are relevant to a specific decision made by a specific decision maker".

As referidas autoras destacam três questões fundamentais para o entendimento deste conceito. A primeira delas é que a qualidade do lucro é condicional à relevância da informação nas decisões. A segunda questão é que a qualidade dos lucros reportados depende se eles são informativos sobre a performance financeira da firma, sabendo que muitos aspectos relativos a performance não são observáveis. E por último, a qualidade do lucro é determinada conjuntamente entre a relevância do desempenho financeiro e a habilidade do sistema contábil em mensurar o desempenho (DECHOW, GE e SCHRAND, 2010).

Portanto, a qualidade do lucro está intimamente ligada com o sistema contábil, que por sua vez é responsável por medir o resultado econômico e financeiro da firma. Nos últimos anos, a contabilidade vem buscando um aperfeiçoamento da norma (GAAP) no sentido de prover informações mais úteis para o processo de decisão dos shareholders e stakeholders. Nesta direção, normas mais baseadas em princípios permitem maior flexibilidade ao gestor para que este possa, por meio de julgamentos, avaliar como representar o efeito econômico das transações vivenciadas pela entidade. 
Por outro lado, a flexibilidade representa um trade-off para a contabilidade contemporânea, pois ao mesmo tempo em que busca uma melhoria na qualidade da informação contábil permite que o gestor tome decisões que não necessariamente representem a realidade da firma, mas sim uma realidade que ele gostaria que fosse. Desta forma o gestor estaria utilizando de práticas discricionárias para gerenciar o resultado.

O tema gerenciamento de resultado não é novo na área de contabilidade e finanças. Muitas são as pesquisas desenvolvidas a respeito do tema, mas ainda existe uma gama de questões em aberto, dada a sua ampla aplicação no setor de negócios. Não existe um consenso sobre o conceito de gerenciamento de resultado, e como não é o foco desta pesquisa discutir qual a melhor ou mais apropriada definição utilizaremos a proposta por Healy e Wahlen (1999, p. 6):

Earnings management occurs when the managers use judgment in financial reporting and in structuring transactions to alter financial reports to either mislead some stakeholders about the underlying economic performance of the company, or to influence contractual outcomes that depend on reported accounting numbers.

O gerenciamento de resultado surge com maior frequência a partir de um conflito de interesse existente dentro da firma entre gestor e acionista. Para Ronen e Yaari (2008) as evidências sugerem que os objetivos entre estes agentes são diferentes em razão de: (i) os gestores e acionistas terem diferentes acessos a privilégios concedidos pela firma e ao mesmo tempo alguns investimentos da firma e decisões de produção imporem custos apenas sobre os gestores; (ii) o portfólio do gestor inclui um capital humano específico da firma, o qual não pode ser aplicado em ambientes diferentes; e (iii) o horizonte de tomada de decisão do gestor é diferente do horizonte do acionista e da firma.

Apesar de todas as decisões terem que ser aprovadas pelo board, o gestor é o responsável por tais decisões, e desta forma adquire um conhecimento específico da firma superior em relação aos demais usuários, inclusive em relação aos acionistas (RONEN e YAARI, 2008). Portanto, é esperado que o gestor, de maneira oportunista, maximize sua utilidade mesmo que isso resulte em prejudicar a qualidade da informação divulgada. Assim, entende-se que quanto maior o nível de gerenciamento de resultado apresentado pela firma, menor será a qualidade do lucro observada nas demonstrações contábeis (SUNDER, 1997).

O gerenciamento de resultados pode ser realizado de duas formas: decisões contábeis e decisões operacionais (CARDOSO e MARTINEZ, 2009). A primeira está associada desde o processo de identificação dos atos e fatos administrativos até a sua evidenciação, passando por critérios de mensuração, classificação e reconhecimento. Já as decisões operacionais representam o distanciamento das práticas operacionais normais da firma, como por exemplo a concessão de desconto de vendas, mudanças no nível de produção etc.

Muitas pesquisas internacionais (JONES, 1991; DECHOW, SLOAN e SWEENEY, 1995; KANG e SIVARAMAKRISHNAN, 1995; BECKER e outros, 1998; DEFOND e PARK, 2001) e nacionais (MARTINEZ, 2008; ALMEIDA e ALMEIDA, 2009; FERREIRA e outros, 2011; PAULO, CAVALCANTE, MELO, 2012) focam o gerenciamento de resultados por intermédio das decisões contábeis, enquanto poucas focam nas decisões operacionais (ROYCHOWDHURY, 2006; COHEN e ZAROWIN, 2010; GUNNY, 2010; ZANG, 2012; CARDOSO e MARTINEZ, 2009).

\section{BOOK-TAX DIFFERENGES}

A diferença existente entre o lucro contábil (book income) e o lucro tributável (taxable income) é usualmente conhecida como book-tax differences. A existência da BTD ocorre em razão de diversos fatores. O mais básico deles é que os sistemas de apuração de lucro seguem diferentes conjuntos de regras, uma vez que seus objetivos não são homogêneos (HANLON e HEITZMAN, 2010).

Enquanto o lucro contábil é calculado com base nos princípios contábeis geralmente aceitos (GAAP), tendo como objetivo principal fornecer ao usuário informação útil para decisões sobre alocação de recurso, o lucro tributável segue os preceitos da legislação tributária e tem por objetivo atender a questões relativas ao governo.

As diferenças geradas podem ser de dois tipos: temporárias ou permanentes. A primeira delas ocorre quando o momento do reconhecimento da receita e/ ou da despesa é diferente entre as normas. Por exemplo: o GAAP orienta o reconhecimento no período $(\mathrm{t})$ e a legislação tributária no período $(t+1)$. A ideia é de que em algum momento no futuro esta diferença será anulada e deixará de ser observada. No que tange à diferença permanente, a questão não é o lapso tempo- 
ral, mas sim o não reconhecimento da receita e/ou da despesa por um dos conjuntos de normas.

Adicionalmente ao fato de que a BTD surge a partir da aplicação imparcial de dois diferentes conjuntos de regras com objetivos distintos, os gestores comumente têm incentivos a aumentar o lucro contábil e reduzir o lucro tributável (WEBER, 2009). Portanto a BTD pode ser composta de dois fatores: (i) diferenças normais provenientes do desalinhamento dos conjuntos de regras contábeis e tributários (NBTD - normal book-tax difference); e (ii) diferenças anormais ocasionadas por práticas discricionárias realizadas pelos gestores ( $A B T D$ - abnormal book-tax difference). As BTDs anormais podem ser oriundas de práticas de gerenciamento sobre o lucro contábil e/ou práticas de gerenciamento sobre o lucro tributável. Dado a maior flexibilidade do conjunto de normas do GAAP em relação às normas tributárias, o gestor tem um maior poder discricionário sobre o lucro contábil do que sobre o lucro tributável (MILLS e NEWBERRY, 2001).

Uma vez que o incentivo do gestor consiste em maximizar o lucro contábil e reduzir o lucro tributável, a BTD pode ser vista como uma proxy de qualidade do lucro. Algumas evidências empíricas na literatura internacional sinalizam nesta direção (GRAHAM, RAEDY e SHACKELFORD, 2012).

Hanlon (2005) identificou que firmas com grande magnitude de BTD apresentam uma baixa persistência dos lucros quando comparada com firmas de baixa $\mathrm{BTD}$, sugerindo desta forma que altos valores de BTD estão relacionados à baixa qualidade de lucro. Adicionalmente, Hanlon (2005) encontra evidências na direção de que o mercado atribui uma baixa expectativa na persistência dos lucros para empresas que demonstram elevadas e positivas BTDs, sugerindo que o investidor observa esta informação como uma "red flag" sobre a qualidade do lucro corrente.

Ayers, Laplante e McGuire (2010) realizaram um estudo sobre a interpretação dos analistas de créditos das informações contidas nas diferenças entre lucro contábil e tributável e observaram que grandes variações positivas e/ou negativas resultam em mudanças menos favoráveis de rating, ou seja, variações na BTD sinalizam uma informação negativa para as agências de rating.

Heltzer (2009) investigou se variações na BTD revelam informações sobre o conservadorismo nos relatórios contábeis subdividindo o conservadorismo em condicional (condicionado a um evento econômico) e incondicional (não condicionado a um evento econômico). Os achados indicam que a relação entre BTD e conservadorismo varia de acordo com a magnitude da BTD. Firmas com extremas BTDs positivas (negativas) são menos (mais) propensas a reconhecer oportunamente perdas (ganhos) nos lucros contábeis, e desta forma apresentam uma baixa qualidade de lucro.

Os resultados de Phillips, Pincus e Rego (2003) indicam a existência de uma relação entre o lucro contábil e o lucro tributável com os incentivos para práticas de gerenciamento de resultado. Adicionalmente os referidos autores encontraram evidências que as despesas tributárias diferidas são uma boa proxy na detecção de gerenciamento de resultados e podem ser utilizada como um suplemento para detectar práticas discricionárias na direção de reduzir ou evitar perdas.

Badertscher e outros (2009) examinaram as implicações tributáveis e não tributáveis do gerenciamento de resultado antes do lucro tributável. Para tanto, os referidos autores subdividiram as práticas de gerenciamento em dois tipos: (i) book-tax conforming (BTC) e (ii) book-tax nonconforming (BTN). A BTC evidencia um gerenciamento de resultado que aumenta ou reduz ambos os lucros, contábil e tributável (por exemplo, real earnings management), enquanto a BTN é um gerenciamento de resultado que aumenta ou reduz apenas o lucro contábil, sem qualquer impacto sobre o lucro tributável, aumentando desta forma a book-tax difference. A pesquisa utilizou uma amostra com firmas que tiveram que reajustar seus resultados para menos em razão de terem gerenciado resultado para mais. Os resultados sinalizam que a predominância do gerenciamento de resultado é o book-tax nonconfirming e, desta forma, que a BTD é uma ferramenta útil para a predição de restatements.

Tang e Firth (2011) investigaram se a BTD era relacionada ao gerenciamento de resultados e à gestão tributária no contexto de empresas chinesas. Os resultados indicaram que o gerenciamento de resultados pode explicar parte do montante das BTDs. De acordo com essa pesquisa, o efeito tributário da BTD seria mais poderoso que o efeito no lucro nos relatórios contábeis.

Comprix, Graham e Moore (2011) analisaram se BTDs mensuradas em nível anual ou mensuradas por variações em séries temporais ao longo de cinco anos estão relacionadas com três formas distintas de divergência de opinião entre os participantes de mercado. Os resultados sugerem que BTDs têm uma associação positiva com relação à turnover de ações, dispersão das previsões dos analistas e com a variância dos retornos.

Observa-se um número restrito de pesquisas desenvolvidas com dados brasileiros. Formigoni, Antunes e Paulo (2009) investigaram se o gerenciamento de 
resultado e/ou o gerenciamento de tributos seriam capazes de explicar a existência da BTD em companhias abertas brasileiras, porém os resultados encontrados foram inconclusivos.

Machado e Nakao (2012) analisaram a utilidade da BTD na identificação do oportunismo existente por parte dos gestores na elaboração das demonstrações financeiras das companhias de capital aberto brasileiras. Os resultados encontrados, para o período de 1994 a 2007, indicam que a média do lucro tributável foi significativamente maior que a média do LAIR, ambos ponderados pelo ativo total.

\section{METODOLOGIA}

Esta pesquisa utilizou duas abordagens para detecção de práticas de gerenciamento de resultados: distribuição de frequências e estimação de accruals discricionários.

A distribuição de frequências foi utilizada para avaliar se há uma descontinuidade em torno do target beating, indicando que haveria gerenciamento de resultados para evitar a apresentação de BTD em nível e/ou variação entre períodos.

No que tange à estimação dos accruals, existem várias maneiras apresentadas pela literatura (JONES, 1991; DECHOW, SLOAN e SWEENEY, 1995; KANG e SILVARAMAKRISHNAN, 1995; DEFOND e PARK 2001). Para fins desta pesquisa foi utilizado o modelo Jones Modificado (DECHOW, SLOAN e SWEENEY, 1995), devido à sua ampla aplicação nas pesquisas em contabilidade e finanças (HEALY e WAHLEN, 1999).

\section{Modelo Jones Modificado}

O Modelo Jones Modificado (DECHOW, SLOAN e SWEENEY, 1995) utiliza a variação das receitas líquidas e dos valores dos ativos imobilizados, partindo do pressuposto de que as acumulações não discricionárias dependem destas variáveis, que são medidas em função dos ativos totais.

O Modelo Jones Modificado busca medir o total de acumulações discricionárias (correntes e não correntes) utilizando as seguintes variáveis, conforme descreve Dechow, Sloan e Sweeney (1995):

$$
N D A_{i t}=\alpha_{i}\left[1 / A_{i t-1}\right]+\beta 1_{i}\left[\Delta R E V_{i t}-\Delta R E C_{i t}\right]+\beta 2_{i}\left[P P E_{i t}\right]+\varepsilon_{i t}
$$
(Equação 1), em que:
- $\quad N D A_{i t}=$ acumulações não discricionárias da firma i no ano t;

- $\Delta R E V_{i t}=$ variação receita bruta da firma i entre os anos t e t-1, ponderados pelos ativos totais no final do período t-1;

- $\Delta R E C_{i t}=$ variação das contas a receber (clientes) da firma i entre os anos t e t-1, ponderados pelos ativos totais no final do período $\mathrm{t}-1$;

- $P P E_{i t}=$ imobilizado da firma i no ano $t$, ponderados pelos ativos totais no final do período t-1;

- $A_{i t-1}=$ ativo total da firma i no ano t-1;

- $\varepsilon_{i t}=$ resíduo da regressão para a firma i no ano t;

As estimativas dos parâmetros da Equação $1 \alpha_{i}, \beta_{1 i}$ e $\beta_{2 i}$ são gerados pelo seguinte modelo:

$T A_{i t} / A_{i t-1}=\alpha_{i}\left[1 / A_{i t-1}\right]+\beta_{1 i}\left[\Delta R E V_{i t}\right]+\beta_{2 i}\left[P P E_{i t}\right]+\varepsilon_{i t}$ (Equação 2), em que:

- $T A_{i t}=$ acumulações totais da firma i no ano t;

- $\quad \varepsilon$ it = resíduo da regressão para a firma i no ano t;

- demais variáveis têm a mesma definição da equação 1.

As acumulações totais são calculadas com base na abordagem do Balanço Patrimonial da seguinte forma:

$T A_{i t}=\left[\left(\Delta A C_{i t}-\Delta D i s p_{i t}\right)-\left(\Delta P C_{i t}-\Delta D i v_{i t}\right)-D e p r i t\right] / A_{i t-1}$ (Equação 3), em que:

- $\Delta A C_{i t}=$ variação do ativo corrente (circulante) da empresa i no final do período t-1 para o final do período t;

- $\Delta$ Disp $p_{i t}=$ variação das disponibilidades da empresa i no final do período t-1 para o final do período t;

- $\Delta P C_{i t}=$ variação do passivo corrente (circulante) da empresa i no final do período t-1 para o final do período t;

- $\Delta D i v_{i t}=$ variação dos financiamentos e empréstimos de curto prazo da empresa i no final do período t-1 para o final do período t;

- $\quad$ Depr $_{i t}=$ montante das despesas com depreciação e amortização da empresa i durante o período t.

- $A_{i t-1}=$ ativo total da firma i no ano t-1;

Os accruals discricionários terão como base de cálculo a diferença entre os totais e os não discricionários, conforme relata Jones (1991, p. 207).

DeAngelo (1986) usou as acumulações totais de um período anterior $(\mathrm{t}-\mathrm{k})$ como uma medida de acumulações totais "normais". Ela define a acumulação "anormal" 
$(\Delta \mathrm{TA})$ como a diferença entre a acumulação total corrente e a acumulação total normal, que, por sua vez, pode ser segregada em discricionária e não discricionária:

$$
T A_{t}=N D A_{t}+D A_{t} \quad(\text { Equação } 4), \text { em que: }
$$

- $D A_{t}=$ acumulações discricionárias da empresa no período t;

- demais variáveis têm a mesma definição das equações 1,2 e 3.

\section{População e amostra}

O espaço temporal contemplado nesta pesquisa foram os exercícios sociais de 2005 a 2009, perfazendo um total de cinco exercícios sociais (informações anuais). Cabe salientar que o exercício social encerrado em 31 de dezembro de 2004 foi utilizado como base de cálculo de variações e serviu de referência para algumas variáveis contempladas no modelo (por exemplo: Ativo Total no período t-1) em relação ao ano de 2005.

O processo de seleção abrangeu inicialmente as empresas de capital aberto brasileiras que atendem pelo menos uma das regras apresentadas abaixo:

- Ser uma empresa membro do IBX100.

- Estar entre as duzentas (200) maiores empresas em volume de vendas em qualquer um dos anos entre 2004 e 2009, conforme informações divulgadas pela Revista Exame.
Após a primeira seleção, foram excluídas as empresas do setor financeiro e as empresas cujas demonstrações financeiras não foram disponibilizadas para a Comissão de Valores Mobiliários (CVM). Urge registrar que tais critérios de exclusão são usuais na literatura internacional e no Brasil.

Como técnica de coleta de dados foram avaliadas as informações contidas no Sistema de Divulgação Externa ITR/DFP/IAN (DIVEXT) disponível no site da Comissão de Valores Mobiliários (CVM). Os dados observados foram os contidos no Balanço Patrimonial, Demonstração do Resultado do Exercício, Notas Explicativas, dentre outros.

As informações contábeis das empresas foram filtradas pelo tipo de Demonstração Consolidada, e caso essas informações não estivessem disponíveis, utilizaram-se dados da controladora.

No que tange especificamente às informações necessárias para a formação do banco de dados, formalizam-se os seguintes parâmetros no Quadro 1:

Após a seleção inicial e alguns ajustes, conforme demonstrado anteriormente, foram analisadas $118 \mathrm{em}$ presas no período de 5 anos, totalizando 485 observações empresas-ano, uma média de 4,11 observações por empresa. Conforme observado no Painel B da Tabela 1, a distribuição da amostra por período é relativamente homogênea. O ano com maior número de observações é o ano de 2009, enquanto o ano com menos observações é o de 2005. A maioria das empresas (64\%) apresenta

\section{Quadro 1 - Especificações de BTD, RTT e NOL}

- 1 - Valor da Conta Resultados Antes da Tributação

Valor encontrado dentro das notas explicativas, especificamente dentro do quadro geralmente intitulado de Reconciliação da alíquota efetiva do imposto de renda e contribuição social sobre o lucro.

- 2 - Lucro Real

Valor calculado a partir da provisão para IR e CSLL (Demonstração do Resultado do Exercício) dividida pela alíquota nominal do IR e CSLL (34\%). Cabe observar que esse cálculo representa uma aproximação do Lucro Real, tendo em vista que essa informação não consta nos informes contábeis.

- 3 - BTD

Valor obtido pela seguinte equação:

(Item 1) - (Item 2)

(Valor da Conta Resultados Antes da Tributação) - (Lucro Real)

- 4 - Regime Tributário de Transição (RTT)

Quando disponível esta informação foi localizada em notas explicativas.

- 5 - Prejuízo Fiscal no ano corrente (NOL)

Quando 0 valor do IR/CSLL corrente for positivo, o NOL será obtido através do referido valor dividido por 0,34. Do contrário, assume-se 0 valor zero, pois houve a ocorrência de Lucro Real. 
informação para todos os períodos analisados, ao passo que apenas $7 \%$ da amostra refere-se a empresas com uma observação. Desta forma, o viés nos resultados que poderia ser gerado por uma amostra não homogênea em relação ao período analisado, bem como pela quantidade de observações por empresas, foi controlado.

\section{Hipóteses de pesquisa}

\section{Hipótese 1}

As empresas gerenciam resultados para aproximar a BTD em nível e em variação do ponto zero.

Supõe-se que as empresas gerenciam resultados para aproximar a BTD em nível e/ou em variação do ponto zero. Mudanças elevadas na diferença entre o lucro contábil e o lucro tributável sinalizam uma baixa qualidade do lucro, e desta forma tais empresas poderiam receber algum tipo de penalização por parte do mercado (HANLON, 2005; AYERS, LAPLANTE e MCGUIRE, 2010; HELTZER, 2009).
Para testar esta hipótese foi utilizada a abordagem de análise de distribuição de frequências, com objetivo de examinar o comportamento estatístico da distribuição entrecruzada (cross-sectional) em torno do ponto zero. Tal metodologia, apesar de suas limitações conclusivas, é largamente utilizada em pesquisa na área de earnings management (BURGSTAHLER e DICHEV, 1997; DEGEROGE, PATEL e ZECKHAISER, 1999; PILCHER e ZAHN, 2008).

\section{Hipótese 2}

A diferença entre o lucro contábil e o lucro tributável (BTD) possui relação com o nível de gerenciamento de resultado.

Supõe-se que a diferença entre o lucro contábil e o lucro tributável (BTD) possui relação com o nível de gerenciamento de resultado. A BTD pode ser composta de dois fatores: (i) diferenças normais provenientes do desalinhamento dos conjuntos de regras contábeis e tributários (NBTD - normal book-tax difference) e (ii)

\section{Tabela 1 - Composição da amostra}

\section{Painel A: Composição da amostra}

\begin{tabular}{|c|c|c|}
\hline Número de empresas investigadas & 118 & \\
\hline Número de anos investigados & 5 & \\
\hline Número de observação (firma-ano) & 485 & \\
\hline Média de observações por empresa & 4,11 & \\
\hline \multicolumn{3}{|c|}{ Painel B: Composição da amostra por ano } \\
\hline Ano & Número de Observações & $\%$ \\
\hline 2009 & 109 & $22 \%$ \\
\hline 2008 & 108 & $22 \%$ \\
\hline 2007 & 96 & $20 \%$ \\
\hline 2006 & 88 & $18 \%$ \\
\hline 2005 & 84 & $17 \%$ \\
\hline Total & 485 & $100 \%$ \\
\hline \multicolumn{3}{|c|}{ Painel C: Composição da amostra - Informações disponiveis (por ano) por empresa } \\
\hline Quantidade de anos & Número de empresas & $\%$ \\
\hline 5 anos & 75 & $64 \%$ \\
\hline 4 anos & 10 & $8 \%$ \\
\hline 3 anos & 11 & $9 \%$ \\
\hline 2 anos & 15 & $13 \%$ \\
\hline 1 ano & 7 & $6 \%$ \\
\hline Total & 118 & $100 \%$ \\
\hline
\end{tabular}


diferenças anormais ocasionadas por práticas discricionárias realizadas pelos gestores (ABTD - abnormal book-tax difference). As BTDs anormais podem ser oriundas de práticas de gerenciamento sobre o lucro contábil e/ ou práticas de gerenciamento sobre o lucro tributável.

Com base em Mills e Newberry (2001) assumimos que o gestor tem um maior poder discricionário sobre o lucro contábil do que sobre o lucro tributável, dada a maior flexibilidade do conjunto de normas do GAAP em relação às normas tributárias. Portanto, existiria uma maior propensão ao gerenciamento ser realizado sobre o lucro contábil. Adicionalmente assumimos por hipótese que o gerenciamento do lucro tributável por escolhas contábeis constituiria fraude fiscal, e desta forma não seria observado.

Com o intuito de testar a hipótese 2, bem como de verificar a relação entre os accruals discricionários e as variáveis de controle, foi realizada uma análise com base no modelo geral para detecção de gerenciamento de resultados contábeis proposto por McNichols e Wilson (1988, p.5):

$$
D A i t=\alpha+\beta\left[P A R T_{i i}\right]+\varepsilon_{i t}(\text { Equação 5), em que: }
$$

- $D A_{i t}=$ accruals discricionários reais da empresa i no período t.

- $\quad P A R T_{i t}=$ conjunto de variáveis particionadas que capturam os fatores que presumidamente motiva o gerenciamento do accruals na empresa i no período t.

- $\quad \varepsilon_{i t}=$ resíduo da regressão para a firma i no ano t;

\section{Hipótese 3}

As empresas com BTD positiva (negativa) estão preponderantemente gerenciando o resultado contábil para mais (menos).

Supõe-se que as empresas com BTD positiva (negativa) estão preponderantemente gerenciando o resultado contábil para mais (menos), uma vez que o gestor estaria aumentado a distância entre o lucro contábil e o lucro tributável. Conforme mencionado anteriormente, assumimos por hipótese que o gerenciamento do lucro tributável por escolhas contábeis constituiria fraude fiscal, e desta forma não seria observado. Assim, assumimos que o distanciamento do lucro contábil em relação ao lucro contábil para mais ou para menos seria alcançado via prática de gerenciamento de resultado, inflando ou deflacionando o lucro contábil na mesma direção.

Para testar a hipótese 3 foram utilizados grupos de controle para analisar o sinal dos accruals discricionários.

\section{RESULTADOS}

Inicialmente são demonstradas as estatísticas descritivas da variável Book-Tax Difference em nível (BTD) e em variação $(\triangle \mathrm{BTD})$, ambas ponderadas pelo Ativo Total no período t-1. Pode-se observar na Tabela 2 que estas variáveis, de acordo com o teste de Jarque-Bera, não seguem uma distribuição normal ao nível de significância de 0,01 .

Os Gráficos 1 e 2 apresentam os histogramas com a distribuição de frequência das variáveis BTD e $\triangle \mathrm{BTD}$. Pode-se observar que a distribuição concentra-se em torno do ponto zero entre o intervalo -0.05 e 0.05 . De acordo com Burgstahler e Dichev (1997), é esperado que os resultados apresentem uma distribuição normal. Desta forma pode-se intuitivamente concluir que existem evidências de um comportamento discricionário para não apresentar resultados de BTD, bem como de $\triangle$ BTD elevados, corroborando inclusive com evidências empíricas anteriores (HANLON, 2005,AYERS, LAPLANTE e MCGUIRE, 2010; HELTZER, 2009).

A análise descritiva das variáveis sugeridas do Modelo Jones Modificado é apresentada no Painel A da Tabela 3. A regressão para estimar os accruals discricionários foi realizada com base na abordagem cross-sectional. Os coeficientes gerados pela regressão

\section{Tabela 2 - Estatística descritiva da variável BTD e $\triangle$ BTD}

\begin{tabular}{|l|c|c|c|c|c|c|c|c|}
\hline Variável & N & Média & Mediana & $\begin{array}{c}\text { Desvio } \\
\text { Padrão }\end{array}$ & Mínimo & Máximo & \multicolumn{2}{|c|}{ Jarque-Bera } \\
\hline BTD & 485 & 0,0149 & 0,0140 & 0,0934 & $-0,4539$ & 1,0545 & $25.709,8300$ & 0,0000 \\
\hline$\triangle$ BTD & 485 & 0,0035 & 0,0008 & 0,1036 & $-0,6440$ & 1,0530 & $16.064,6200$ & 0,0000 \\
\hline
\end{tabular}


(Equação 2) são apresentados no Painel B do Tabela 3. As variáveis $1 / A_{i t-1}, \triangle R E V_{i t}$ e $P P E_{i t}$ apresentaram significância estatística ao nível de 0,01. Apenas a constante do modelo não demonstrou ser significativamente diferente de zero.

O modelo estimado apresenta limitações de heterocedasticidade, autocorrelação serial e de não normalidade dos resíduos. Cumpre destacar que com base no
Teorema Central do Limite os estimadores do método de Mínimos Quadrados Ordinários satisfazem a normalidade assintótica, ou seja, em amostras suficientemente grandes eles se aproximam de uma distribuição normal (WOOLDRIDGE, 2005). Portanto, apesar de teste específico, o pressuposto de normalidade é relaxado no que tange aos parâmetros do modelo, pois seus coeficientes estimados são consistentes e não viesados

\section{Gráfico 1 - Distribuição de frequência BTD}

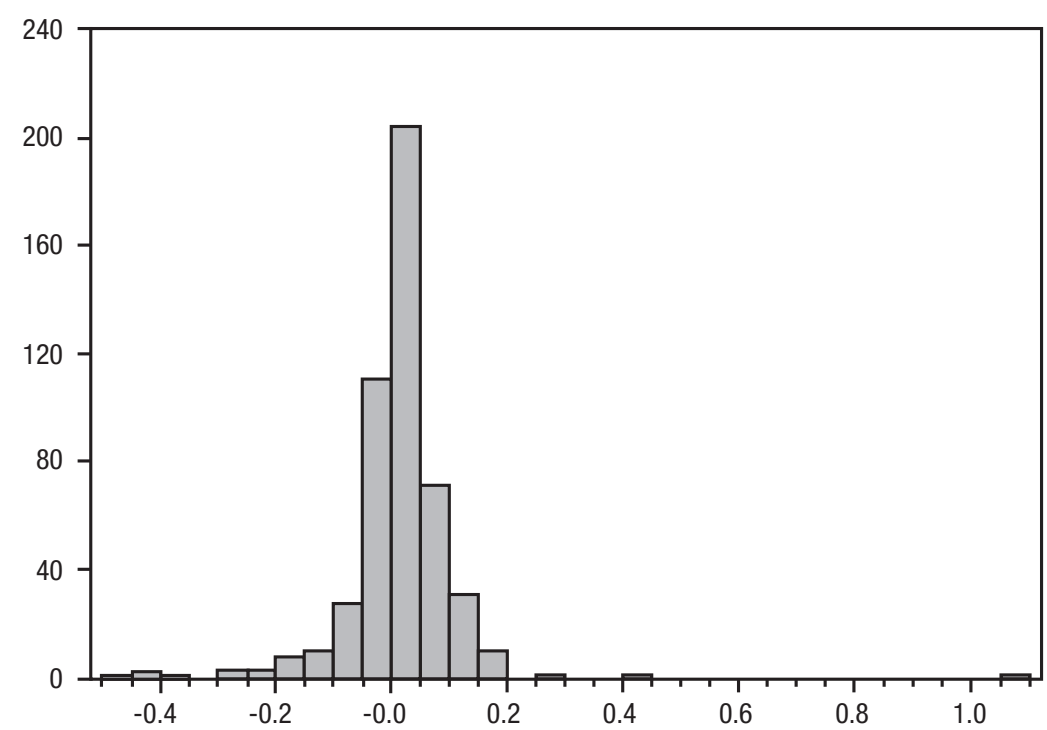

\section{Gráfico 2 - Distribuição de frequência $\triangle B T D$}

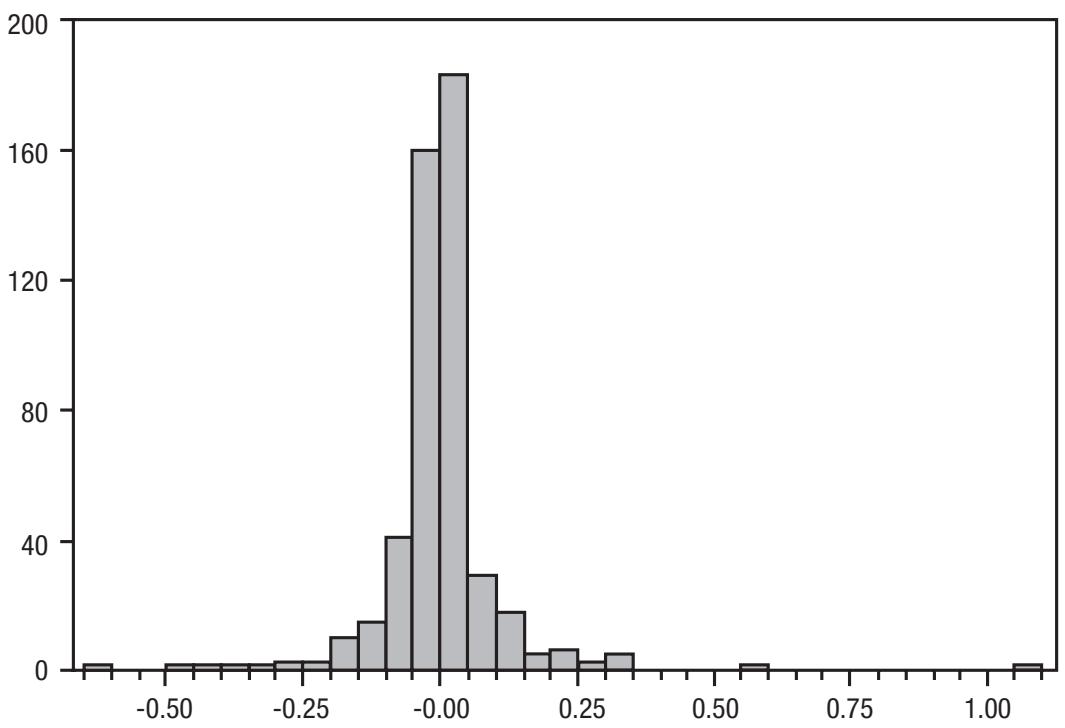


assintoticamente, ainda que sob a presença de heterocedasticidade e autocorrelação (FORMIGONI, ANTUNES e PAULO, 2009).

No caso específico da hipótese 2, o objetivo é verificar se a BTD possui relação com o nível dos accruals discricionários, independente se para mais ou para menos. Portanto, optou-se por trabalhar com o valor absoluto dos accruals discricionários e da BTD. Adicionalmente foram aplicados controles do tipo: (i) alteração na norma contábil, proveniente da Lei n. 11.638/07; (ii) opção pelo regime tributário de transição; (iii) apuração de prejuízo fiscal no ano corrente; e (iv) tamanho da firma por meio do logaritmo natural do ativo.
$D A(A B S)=\alpha+\beta_{1}\left[B T D \_A B S\right]+\beta_{2}[A N O]+\beta_{3}[R T T]+$
$\beta_{4}[N O L]+\beta_{5}\left[L N \_A T\right]$

- $D A(A B S)=$ accruals discricionários na forma absoluta;

- $\quad B T D \_A B S=$ diferença entre o lucro contábil e o lucro tributável (BTD) na forma absoluta;

- $\quad A N O=$ variável dummy (1 = ano posterior à Lei $\mathrm{n}$. $11.638 / 07$, se não $=0)$;

- $\quad$ RTT = variável dummy $(1=$ Regime Tributário de Transição, se não = 0);

- $\quad N O L=$ variável dummy $(1=$ Prejuízo Fiscal no ano corrente, se não $=0$ );

- $\quad L N \_A T=$ logaritmo natural da variável ativo total;

\section{Tabela 3 - Estatística descritiva e estimativa dos coeficientes Modelo Jones Modificado}

\begin{tabular}{|l|c|c|c|c|c|c|}
\hline \multicolumn{7}{|c|}{ Painel A: Estatística Descritiva variáveis modelo Jones Modificado } \\
\multicolumn{1}{|c|}{ Variável } & N & Média & Mediana & Desvio Padrão & Mínimo & Máximo \\
\hline $\mathrm{TA}_{\mathrm{it}}$ & 485 & $-0,0301$ & $-0,0408$ & 0,1371 & $-0,5494$ & 1,0370 \\
\hline $1 / \mathrm{A}_{\mathrm{it}-1}$ & 485 & $4,37 \mathrm{E}-07$ & $2,90 \mathrm{E}-07$ & $5,58 \mathrm{E}-07$ & $3,42 \mathrm{E}-09$ & $6,52 \mathrm{E}-06$ \\
\hline$\Delta \mathrm{REV}_{\mathrm{it}}$ & 485 & 0,1246 & 0,0631 & 0,4335 & $-6,0851$ & 3,9293 \\
\hline$\triangle \mathrm{REC}_{\mathrm{it}}$ & 485 & 0,0226 & 0,0091 & 0,0701 & $-0,3311$ & 0,4702 \\
\hline $\mathrm{PPE}_{\mathrm{it}}$ & 485 & 0,4900 & 0,4826 & 0,3179 & 0,0000 & 2,6241 \\
\hline
\end{tabular}

Obs: todas as variáveis do Painel A não seguem distribuição normal com base no teste de Jarque-Bera

Painel B: Estimativa coeficientes modelo Jones Modificado

\begin{tabular}{|c|c|c|c|c|c|c|}
\hline \multirow{2}{*}{ Variável } & \multicolumn{6}{|c|}{ Jones Modificado } \\
\hline & \multicolumn{2}{|c|}{ Coeficientes } & \multicolumn{2}{|c|}{ Desvio Padrão } & Estatistica-t & Valor-p \\
\hline Constante & \multicolumn{2}{|c|}{$-0,0118$} & \multicolumn{2}{|c|}{0,0118} & $-0,9986$ & 0,3185 \\
\hline $1 / A_{i t-1}$ & \multicolumn{2}{|c|}{47598,7200} & \multicolumn{2}{|c|}{10579,6700} & 4,4991 & 0,0000 \\
\hline$\triangle \mathrm{REV}_{\text {it }}$ & \multicolumn{2}{|c|}{0,0561} & \multicolumn{2}{|c|}{0,0137} & 4,0972 & 0,0000 \\
\hline $\mathrm{PPE}_{\mathrm{it}}$ & \multicolumn{2}{|c|}{$-0,0940$} & \multicolumn{2}{|c|}{0,0186} & $-5,0457$ & 0,0000 \\
\hline $\mathrm{R}^{2}$ & \multicolumn{2}{|c|}{0,1157} & & & & \\
\hline $\mathrm{R}^{2}$ ajustado & \multicolumn{2}{|c|}{0,1102} & & & & \\
\hline F-statistic & \multicolumn{2}{|c|}{20,9750} & & & & \\
\hline Prob (F-statistic) & \multicolumn{2}{|c|}{0,0000} & & & & \\
\hline Durbin-Watson & \multicolumn{2}{|c|}{1,9809} & & & & \\
\hline White Heteroskedasticity Test & \multicolumn{2}{|c|}{21,1305} & & & & \\
\hline Breusch-Goldfrey Serial Correlation LM Test & \multicolumn{2}{|c|}{3,3422} & & & & \\
\hline \multicolumn{7}{|c|}{ Painel C: Estatística Descritiva das acumulações discricionárias estimadas pelo modelo Jones Modificado } \\
\hline Variável & N & Média & Mediana & Desvio Padrão & Mínimo & Máximo \\
\hline $\mathrm{DA}_{\text {it }}$ & 485 & 0,0013 & $-0,0010$ & 0,1309 & $-0,4885$ & 0,9261 \\
\hline $\mathrm{DA}_{\mathrm{it}}$ (sinal positivo) & 240 & 0,0852 & 0,0464 & 0,1164 & 0,0003 & 0,9261 \\
\hline $\mathrm{DA}_{\mathrm{it}}$ (sinal negativo) & 245 & $-0,0809$ & $-0,0567$ & 0,0837 & $-0,4885$ & $-0,0002$ \\
\hline
\end{tabular}


De acordo com o Painel B da Tabela 4 é possível observar que as variáveis BTD_ABS e LN_AT apresentam-se estatisticamente significante ao nível de 0,05 e a variável RTT estatisticamente significante ao nível de 0,10, para explicar os accruals discricionários na forma absoluta. Desta forma, a hipótese 2 de que as diferenças entre o lucro contábil e o lucro tributável (BTD) possui relação com o nível de gerenciamento de resultado foi validada. O sinal positivo do coeficiente da variável BTD _ABS indica que quanto maior o nível absoluto de BTD maior será o montante na forma absoluta das acumulações discricionárias. No que tange ao tamanho da firma, controlado pela variável LN_AT, foram encontradas evidências da existência de uma relação inversamente proporcional aos accruals totais, ou seja, que quanto maior o tamanho da firma menor o nível de práticas discricionárias sobre o lucro da firma. Algumas justificativas podem ser sugeridas para tal evidência, como melhores níveis de ferramentas de governança coorporativa, auditoria, monitoramento etc, porém não faz parte do escopo desta pesquisa identificar quais os reais motivos para tal evidência. Quanto às firmas que adotaram o regime tributário de transição, concluímos que, com base no sinal negativo do coeficiente estimado, gerenciam menos o resultado do que as que não adotaram tal regime.

As demais variáveis testadas neste trabalho não explicam a variável DA (ABS). Portanto, não foram encontrada evidências de que a adoção da Lei n. 11.638/07 tenha influenciado o nível das acumulações discricionárias no

\section{Tabela 4 - Estatística descritiva e estimativa dos coeficientes análise multivariada}

\begin{tabular}{|l|c|c|c|c|c|c|}
\hline \multicolumn{7}{|l|}{ Painel A: Estatística Descritiva variáveis modelo Jones Modificado } \\
\hline Variável & N & Média & Mediana & Desvio Padrão & Mínimo & Máximo \\
\hline DA (ABS) & 485 & 0,0830 & 0,0510 & 0,1012 & 0,0002 & 0,9261 \\
\hline BTD_ABS & 485 & 0,0544 & 0,0352 & 0,0773 & 0,0000 & 1,0545 \\
\hline ANO & 485 & 0,4474 & 0,0000 & 0,4977 & 0,0000 & 1,0000 \\
\hline RTT & 485 & 0,0351 & 0,0000 & 0,1841 & 0,0000 & 1,0000 \\
\hline NOL & 485 & 0,0371 & 0,0000 & 0,1892 & 0,0000 & 1,0000 \\
\hline LN_AT & 485 & 15,4137 & 15,1626 & 1,2326 & 12,6525 & 19,6608 \\
\hline
\end{tabular}

Obs: todas as variáveis do Painel A não seguem distribuição normal com base no teste de Jarque-Bera

\section{Painel B: Estimativa dos coeficientes análise multivariada}

\begin{tabular}{|l|c|c|c|c|}
\hline \multicolumn{1}{|c|}{ Variável } & \multicolumn{3}{|c|}{ Jones Modificado } \\
\hline Constante & Coeficientes & Desvio Padrão & Estatistica-t & Valor-p \\
\hline BTD_ABS & 0,2767 & 0,0564 & 4,9080 & 0,0000 \\
\hline ANO & 0,2206 & 0,0587 & 3,7610 & 0,0002 \\
\hline RTT & 0,0067 & 0,0092 & 0,7331 & 0,4638 \\
\hline NOL & $-0,0416$ & 0,0245 & $-1,6989$ & 0,0900 \\
\hline LN_AT & $-0,0025$ & 0,0237 & $-0,1059$ & 0,9157 \\
\hline R2 & $-0,0134$ & 0,0036 & $-3,6932$ & 0,0002 \\
\hline R2 ajustado & 0,0641 & & & \\
\hline F-statistic & 0,0544 & & & \\
\hline Prob(F-statistic) & 6,5648 & & & \\
\hline Durbin-Watson & 0,0000 & & & 0,5777 \\
\hline White Heteroskedasticity Test & 1,8904 & & \\
\hline Breusch-Goldfrey Serial Correlation LM Test & 2,4394 & & \\
\hline
\end{tabular}


período estudado. Da mesma forma não foram encontradas evidências de que a apuração de um prejuízo fiscal no ano corrente tenha influenciado a utilização de práticas discricionárias por parte dos gestores.

Para testar a hipótese 3 de que as empresas com BTD positiva (negativa) estão preponderantemente gerenciando o resultado contábil para mais (menos), a amostra foi segregada em dois grupos de acordo com o sinal da BTD ponderada pelo ativo total no período t-1. Para segregação da amostra nestes dois grupos foi utilizada uma variável dummy denominada S_BTD (em que: $0=$ BTD com sinal negativo e 1 = BTD com sinal positivo).

Pode-se observar que no grupo BTD (positiva) o predomínio dos accruals discricionários foi de sinal positivo, ou seja, a maior quantidade de empresas utilizou práticas de gerenciamento para inflar seus lucros. Tais resultados estão relacionados à hipótese 3, corroborando que o sinal da BTD e dos accruals discricionários seguem preponderantemente na mesma direção. A mesma evidência é encontrada quando analisadas as empresas com BTD (negativa), as quais apresentaram accruals discricionários preponderantemente e em média negativos.

\section{CONCLUSÃO}

Este trabalho buscou evidências de associação entre práticas de gerenciamento de resultados e a diferença entre o lucro contábil e lucro tributável (BTD). Foi possível diagnosticar que as companhias de capital aberto brasileiras buscam apresentar o montante de BTD em nível e em variação em torno do ponto zero e desta forma evitar algum tipo de penalização por parte do mercado, dado que elevados valores e oscilações da BTD estão associados à baixa qualidade do lucro.

Em relação ao nível de gerenciamento de resultado com o montante da BTD foi identificada uma relação diretamente proporcional, ou seja, quanto maior o valor da BTD em termos absolutos, maior o valor dos accruals discricionários. Este resultado sugere que a BTD pode servir de suplemento para detectar práticas discricionárias utilizadas pelos gestores. Adicionalmente foram encontradas evidências de que o tamanho da firma e a adoção do regime tributário de transição estão inversamente proporcionais relacionadas ao nível das acumulações discricionárias.

No que tange à mudança proporcionada pela promulgação da Lei n. 11.638/07, a qual trouxe uma maior flexibilidade para mensuração das variações patrimoniais, não foi identificada relação desta com os accruals discricionários. O estudo também não identificou associação entre a apuração de prejuízos fiscais no ano corrente com práticas de gerenciamento de resultado.

A análise dos dados com base nos grupos de empresa com BTD (positiva) e BTD (negativa) forneceu evidências de que as entidades preponderantemente gerenciam seus resultados na mesma direção do sinal observado da BTD.

Os dados permitiram expandir os achados empíricos sobre práticas de gerenciamento de resultados e BTD. Em relação à literatura internacional, este estudo pode ser visto como complementar, uma vez que reafirmou a maioria das evidências encontradas em ambientes externos, ao passo que do ponto de vista da literatura nacional este trabalho tem relevância significativa, pois pesquisas empíricas contemplando dados brasileiros até o momento da realização deste estudo eram escassas e apresentavam resultados inconclusivos.

Cumpre informar que os achados deste trabalho restringem-se às entidades pesquisadas, bem como ao intervalo temporal objeto desta pesquisa. Adicionalmente, este estudo apresenta limitações referentes ao modelo estatístico utilizado.

Por se tratar de uma literatura relativamente recente no Brasil, sugere-se um aprofundamento do tema, por

Tabela 5 - Acumulações discricionárias por sinal de BTD

\begin{tabular}{|c|c|c|c|c|}
\hline & \multicolumn{2}{|c|}{ BTD (positiva) } & \multicolumn{2}{|c|}{ BTD (negativa) } \\
\hline & $\mathbf{N}$ & $\%$ & $\mathbf{N}$ & $\%$ \\
\hline D.A. positivas & 179 & $56 \%$ & 61 & $37 \%$ \\
\hline D.A. negativas & 139 & $44 \%$ & 106 & $63 \%$ \\
\hline Total & 318 & $100 \%$ & 167 & $100 \%$ \\
\hline Média (DA) & \multicolumn{2}{|c|}{0,0194} & \multicolumn{2}{|c|}{$-0,0332$} \\
\hline Desvio Padrão & \multicolumn{2}{|c|}{0,1305} & \multicolumn{2}{|c|}{0,1250} \\
\hline
\end{tabular}


meio de pesquisas futuras, de questões não contempladas neste trabalho. Uma análise cross-country (segregando países code law e common law) permitiria evidenciar se a relação entre BTD e earnings management segue em linha com os resultados evidenciados no mercado brasileiro ou se apresenta diferentes níveis, bem como se está relacionada a diferentes tipos de incentivos. Adicionalmente, seria de grande relevância estudos que explorassem não só o impacto do gerenciamento de resultado no mercado acionário (por exemplo, efeito no preço da ação, bem como volume de negócios), mas também a resposta do investidor a diferentes níveis de BTD. Por fim , a extensão do espaço temporal, bem como a aplicação de novos modelos econométricos seriam interessantes no intuito de aumentar a robustez dos achados.

\section{NOTA DA REDAÇÃO}

Este artigo foi apresentado no XXXV Encontro da Associação Nacional de Pós-Graduação e Pesquisa em Administração (EnANPAD) realizado em 2011 e convidado a participar do processo de avaliação double blind review da RAE pela Diretoria da ANPAD.

\section{REFERÊNCIAS}

AYERS, B; JIANG, J; YEUNG, P. Discretionary Accruals and Earnings Management: An analysis of pseudo earnings target. The Accounting Review, v. 81, n. 3, p. 617-652, 2006.

AYERS, B; LAPLANTE S; MCGUIRE, S. Credit rankings and taxes: the effect of book/tax differences on ratings changes. Contemporary Accounting Research, v. 27, n. 2, p. 359-402, 2010.

ALMEIDA, J. E; ALMEIDA, J. C. Auditoria e earnings management: estudo empírico nas empresas abertas auditadas pelas big four e demais firmas de auditoria. Revista Contabilidade \& Finanças, v. 20, n. 50, 2009.

BADERTSCHER, B. A. e outros. Earnings Management Strategies and the Trade-Off between Tax Benefits and Detection Risk: To Conform or Not to Conform? The Accounting Review. v. 84, n. 1, p. 63-97, 2009.

BECKER e outros. The effect of audit quality on earnings management. Contemporary Accounting Research. Toronto, v. 15, n. 1, p. 1-24, 1998.
BRASIL. Lei n. 11.638, de 28/12/2007. Diário Oficial da República Federativa do Brasil, Poder Executivo, Brasília, DF, 28/12/2007. Secção 1, p. 2.

BRASIL. Lei n. 11.941, de 27/05/2009. Diário Oficial da República Federativa do Brasil, Poder Executivo, Brasília, DF, 28/05/2009. Secção 1, p. 3.

BURGSTAHLER, D. C; DICHEV, I. Earnings management to avoid earnings decrease and losses. Journal of Accounting and Economics. New York, v. 24, n.1, p.99-126, 1997.

CARDOSO, R. L; MARTINEZ, A. L. Gerenciamento da informação contábil no Brasil mediante decisões operacionais. Revista Eletrônica de Administração, v. 15, n. 3, p. 1-27, 2009.

COHEN, D. A; ZAROWIN, P. Accrual-based and earnings management activities around seasoned equity offerings. Journal of Accounting and Economics, v.. 50, n.1, p. 2-19, 2010

COMPRIX, J; GRAHAM, R. C; MOORE, J. A. Empirical Evidence on the Impact of Book-Tax Differences on Divergence of Opinion among Investors. Journal of the American Taxation Association. v. 33, n. 1, p. 51-78, 2011.

DEANGELO, L. E. Accounting Numbers as Market Valuation Substitutes: A Study of Management Buyouts of Public Stockholders. The Accounting Review, v. 61, n. 3, p. 400420, 1986.

DECHOW, P. M; SLOAN, R. G; SWEENEY, A. P. Detecting Earnings Management. The Accounting Review, v. 70, n. 2, p. 193-225, 1995.

DECHOW, P; GE, W; SCHRAND, C. Understanding Earnings Quality: A review of the proxies, their determinants and their consequences. Journal of Accounting and Economics, v. 50, n. 3, p. 344-401, 2010.

DEFOND, M. L; PARK, C. W. The Reversal of Abnormal Accruals and the Market Valuation of Earnings Surprises. The Accounting Review, v. 76, n. 3, p. 375-404, 2001.

DEGEROGE F; PATEL J; ZECKHAISER, R. Earnings management to exceed thresholds. Journal of Business, Chicago, v. 772, n. 1, p. 1-33, 1999.

FERREIRA, F.R. ; CARDOSO, R. L. ; MARTINEZ, A. L. ; MÁRIO, P. C. . Auditing and Earnings Management in Brazilian HMOs. Corporate Ownership \& Control, v. 8, n. 3, p. 632-644, 2011. 
FORMIGONI, H; ANTUNES, M. T; PAULO, E. Diferença entre o lucro contábil e lucro tributável: uma análise sobre o gerenciamento de resultados contábeis e gerenciamento tributário nas companhias abertas brasileiras. BBR Brazilian Business Review, v. 6, n. 1, p.44-61, 2009.

GRAHAM, J.R; RAEDY, J.S; SCHACKELFORD, D.A. Research in accounting for income taxes. Journal of Accounting and Economics, v. 53, n. 1-2, p. 412-434, 2012,

GUNNY, K. The relation between earnings management using real activities manipulation and future performance: evidence from meeting earnings benchmarks. Contemporary Accounting Research, v. 27, n. 3, p. 855-888, 2010.

HANLON, M. The persistence and pricing of earnings, accruals, and cash flows when firms have large book-tax differences. The Accounting Review, v. 80, n. 1, p. 137-166, 2005.

HANLON, M; HEITZMAN, S. A Review of Tax Research. Journal of Accounting and Economics, n. 2, p. 127-178, 2010.

HEALY, P. M; WAHLEN, J. M. A review of the earnings management literature and its implications for standard setting. Accounting Horizons, v. 13, n. 4, p. 365-384, 1999.

HELTZER, W. Conservatism and book-tax differences. Journal of Accounting, Auditing and Finance, v. 24, n. 3, p. 469-504, 2009.

JONES, J. J. Earnings management during import relief investigations. Journal of Accounting Research, v. 29, n. 2, p. 193-228, 1991.

KANG, S; SIVARAMAKRISHNAN, K. Issues in testing earnings management and an instrumental variables approach. Journal of Accounting Research, v. 33, n. 2, p. 353$367,1995$.

MACHADO, M. C.; NAKAO, S. H., Diferenças entre o lucro tributável e o lucro contábil das empresas brasileiras de capital aberto. Revista Universo Contábil, v. 8, n. 3, 2012.

MARTINEZ, A. L. Detectando Earnings management no Brasil: estimando os accruals discricionários. Revista Contabilidade E Finanças, v. 19, n. 46, p. 7-17, 2008.

MCNICHOLS, M; WILSON G. P. Evidence of earnings management from the provision for bad debts. Journal of Accounting Research, v. 26, n. 3, p. 1-31, 1988.
MILLS, L; NEWBERRY, K. The Influence of Tax and Nontax cost on Book-tax Reporting Difference: Public and Private Firms. The Journal of the American Taxation Association, v. 23, n. 1, p. 1-19, 2001.

PAUlO, E; CAVAlCANTE, P. R; MELO, I. I. Qualidade das informações contábeis na oferta pública de ações e debêntures pelas companhias abertas brasileiras. Brazilian Business Review, v. 9, n. 1, p. 1-26, 2012.

PILCHER, R; ZAHN, J-L.W. M. V. Earnings Management and Income Distribution Around a 'Breakeven or Near Breakeven Surplus' Benchmark with the Public Sector. In: AFAANZ Annual Conference, Sydney, 2008.

PHILliPS, J; PINCUS, M; REGO, S. O. Earnings Management: New Evidence Based on Deferred Tax Expense. The Accounting Review, v. 78, n. 2, p. 491-521, 2003.

RONEN, J; YAARI, V. Earnings Management Emerging Insights in Theory, Practice, and Research. Springer, New York, 2008.

ROYCHOWDHURY, S. Earnings Management through real activities manipulation. Journal of Accounting and Economics, v. 42, n. 3, p. 335-370, 2006.

SHACKELFORD, D; SHEVLIN, T. Empirical tax research in accounting. Journal of Accounting and Economics, v. 31, n. 2, p. 321-387, 2001.

SUNDER, S. Theory of accounting and control. Cincinnati: South-Western Publishing, 1997.

TANG, T; FIRTH, M. Can Book-Tax Differences Capture Earnings Management and Tax Management? Empirical Evidence from China. The International Journal of Accounting, vol. 46, n. 2, p. 175-204, 2011

WEBER, D. Do analysts and investors fully appreciate the implications of book-tax differences for future earnings? Contemporary Accounting Research, v. 26, n. 4, p. 11751206, 2009.

WOOLDRIDGE, J. Introductory Econometrics. South-Western Publishing, 2005.

ZANG, A. Y. Evidence on the tradeoff between real manipulation and accruals manipulation. The Accounting Review, v.87, p. 675-703, 2012. 